\title{
Propuesta de mejora en la calidad del aire: caso Zona Metropolitana de Guadalajara
}

\author{
Joel García Galván ${ }^{1}$ \\ Martin G. Romero MoretT ${ }^{2}$ \\ Ma. Magdalena VelázQuez Contreras ${ }^{3}$
}

\section{Resumen}

Este ensayo tiene como objetivo analizar los diversos escenarios en las actividades económicas y la relación que se da entre el Sector Público y el Sector Privado, al proponer trabajar de manera coordinada en un proyecto de inversión con enfoque medioambiental. Para este caso, sobre una disminución en la contaminación del aire en una parte de la Zona Metropolitana de Guadalajara (Jalisco, México). Con base a la información vista en el Seminario "Prospectiva, pensamiento complejo y transdisciplina", y como parte de las actividades indicadas, se elaboró material didáctico que ejemplifica el desarrollo de conceptos, metodología y herramientas económicas y financieras de análisis utilizadas en la materia de Formulación y Evaluación de Proyectos impartida en la Licenciatura de Economía de la Universidad de Guadalajara. Se utiliza la metodología de marco lógico, en la que se articula la relación entre el Sector Público y el Sector Privado, como vínculos que se dan de manera sistémica ante un entorno complejo, como es la contaminación del aire en las ciudades y que afecta la salud pública de la población. En los resultados se presenta el análisis de las decisiones e indicadores dados por instituciones y dependencias gubernamentales, se plantea la viabilidad del proyecto y la rentabilidad y se concluye que, debido al proce-

Fecha de Recepción: 18 de octubre de 2020 Fecha de Aceptación: 10 de diciembre de 2020.

1 Profesor Inv. Asociado B. Departamento de Economía. Miembro del UDG-CA-116 “Teoría Económica y Desarrollo Sustentable" Centro Universitario de Ciencias Económico Administrativas (CUCEA). Universidad de Guadalajara. Correo electrónico: joel.garcia@cucea.udg.mx

2 Profesor Inv. Titular C. Departamento de Economía. Miembro del UDG-CA-116 "Teoría Economica y Desarrollo Sustentable" CUCEA. Universidad de Guadalajara.

3 Licenciada en Educación. Universidad Virtual (UDG) y Candidata a Maestra. Maestría en Políticas de la Educación Superior (CUCEA-UDG) Correo electrónico: maestriamagda@gmail.com 
so electoral se deberá consensar la disponibilidad y uso de los recursos económicos, dando prioridad a: la salud, la economía y el medioambiente.

Palabras clave: educación, salud, medioambiente.

\section{Resume}

This essay aims to analyze the various scenarios in economic activities and the relationship that exists between the Public Sector and the Private Sector, by proposing to work in a coordinated manner on an investment project with an environmental focus. For this case, about a decrease in air pollution in a part of the Guadalajara Metropolitan Area (Jalisco, México). Based on the information seen in the Seminar: "Prospective, complex thinking and transdiscipline", and as part of the activities indicated, didactic material was developed that exemplifies the development of concepts, methodology and financial analysis tools used in the subject of Formulation and Evaluation of Projects taught in the Economics Degree of the University of Guadalajara. The logical framework methodology is used, in which the relationship between the Public Sector and the Private Sector is articulated, as links that occur in a systemic way in a complex environment, such as air pollution in cities and that affects the public health of the population. In the results, the analysis of the decisions and indicators given by institutions and government agencies is presented, the viability of the projects and the profitability are considered and it is concluded that, due to the electoral process, the availability and use of economic resources must be agreed upon, giving priority to: health, economy and environment.

Keywords: education, health, environment.

\section{Introducción}

El proyecto como respuesta a un problema social para la asignatura de Formulación y Evaluación de Proyectos, llevada en la Licenciatura de Economía de la Universidad de Guadalajara.

Los valores que alcanzan los indicadores de la "variable problema" al momento de su identificación es la línea base. Es deseable que la identificación y caracterización técnica de los problemas sociales sean equivalentes a las demandas efectivas de la población. No se debe confundir un problema social con la ausencia de cobertura o falta de entrega de un servicio específico. Circunscribir el problema social a la gestión, calidad o cantidad de la oferta existente limita el campo de intervención y la posibilidad de alcanzar los objetivos perseguidos.

Por ello, como herramienta que "permite estructurar los procesos esenciales de un proyecto como la conceptualización, el diseño, el seguimiento de la ejecución y evaluación" (OBS Business School, s/f), utilizaremos la metodología de marco lógico para intervenir en un problema medioambiental, como es la contaminación del aire a causa de la generación de Co2. 
La metodología del marco lógico, como un sistema de procedimientos e instrumentos para una planificación de proyectos orientada a objetivos (Agencia de cooperación alemana para el desarrollo [GTZ], 1987). Esta metodología con su árbol de objetivos y su árbol de problemas, ayuda a la clarificación del proceso de planificación del proyecto en todas sus etapas, así como a comunicar información esencial sobre el mismo.

En el caso de la técnica del árbol de problemas, se identifica un problema central para posteriormente analizar las problemáticas que de éste se derivan y las relaciones causa-efecto entre sí mismas (UNESCO, 2017). Se presenta a continuación el esquema 1: Árbol de problemas.

\section{Esquema 1}

Árbol de problemas

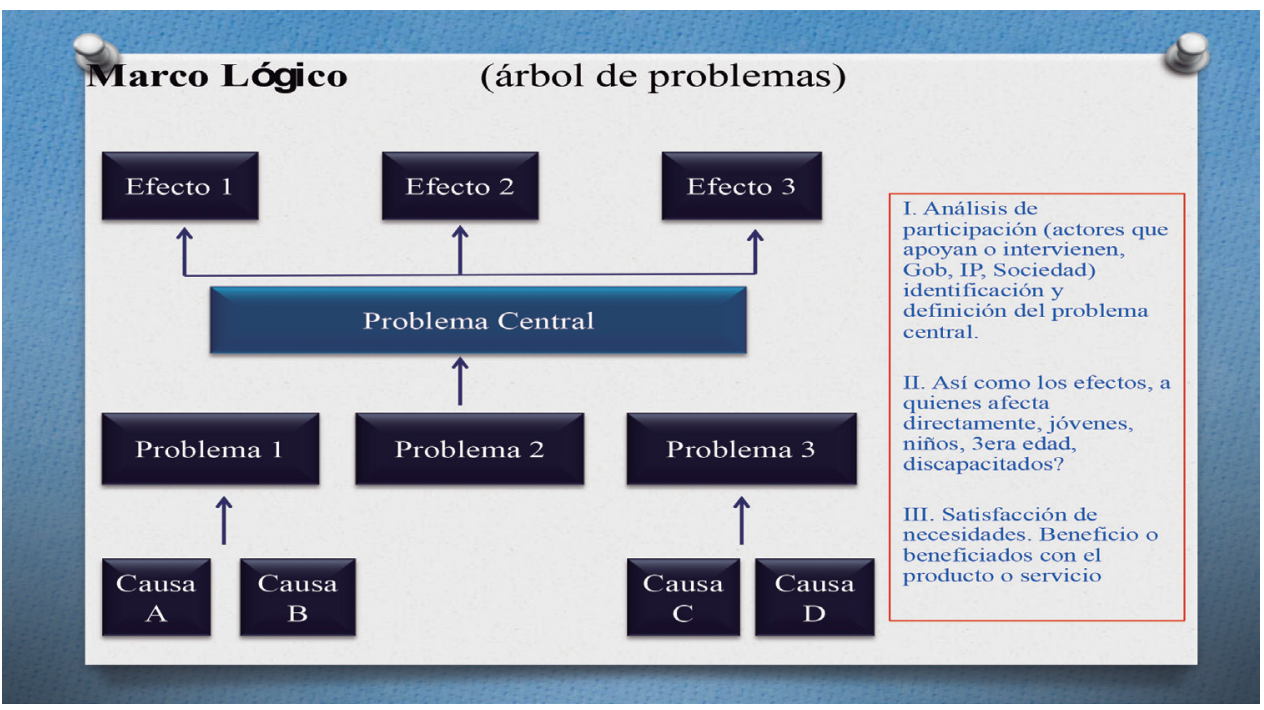

Fuente: Elaboración propia.

Una vez elaborado el árbol de problemas, se analizarán las posibles soluciones a cada una de las problemáticas, de manera que éste se transforme en el árbol de objetivos, mismos que se analizarán para determinar las estrategias a seguir. 


\section{Esquema 2}

Árbol de objetivos

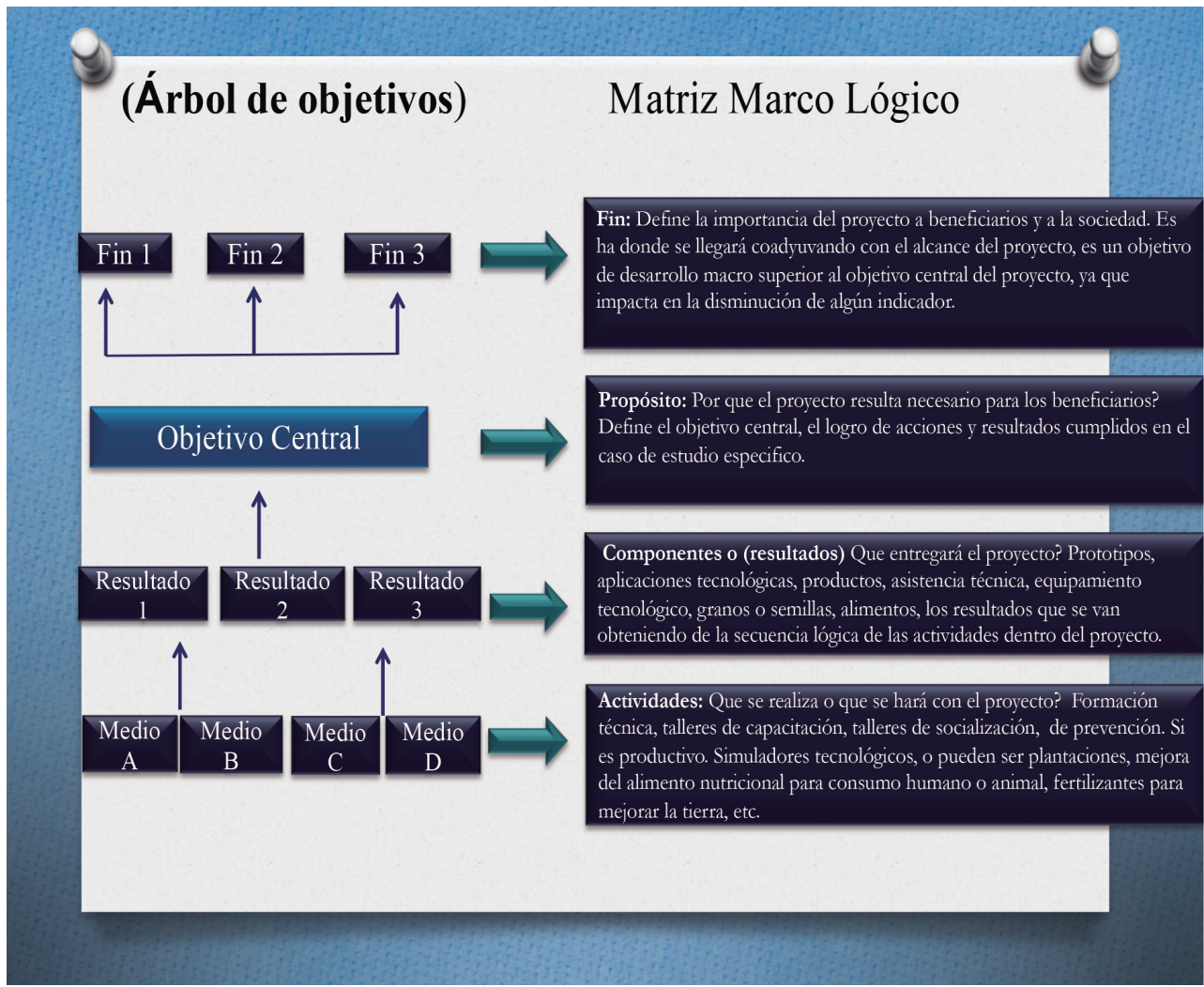

Fuente: Elaboración propia.

\section{¿Qué son los Medios de verificación?}

Son las evidencias: Conceptos: los indicadores y medios de verificación deben ser prácticos y económicos (también la inversión puede ser a fondo perdido y se debe administrar el recurso económico escaso y con la certeza de alcanzar el máximo beneficio social). No tener subejercicios ni desvíos. Los medios de verificación proporcionan las bases para supervisar y evaluar el proyecto.

\section{¿Qué son los Supuestos?}

Cada proyecto comprende riesgos ambientales, financieros, institucionales, sociales, políticos, climatológicos u otros factores que pueden hacer que el mismo fracase. La 
matriz de marco lógico requiere que el equipo de diseño de proyecto identifique los riesgos en cada etapa: actividad, componente, propósito y fin. Los supuestos deben situarse en el nivel indicado de acuerdo a su planteamiento. Deben ser realistas y adecuados al contexto del proyecto. Proporcionar las bases para supervisar y evaluar el proyecto.

\section{Propuesta para la mejora de la calidad del aire. \\ Ejemplo: Problema central por contaminacion Co2 \\ Objetivo central, proyecto vereficentro vehicular. \\ Material didactico.}

\section{Antecedente:}

Según las estaciones de monitoreo ambiental llevamos 20 años contaminando de forma ascendente. En 2013 uno de cada tres días del año (123) fueron de mala calidad en la ciudad repercutiendo en la salud de 4.6 millones de habitantes en la ZMG. Según SEMADET se estima que $85 \%$ de las emisiones a la atmosfera son causa de los automotores.

Según cifras de la Organización Mundial de la Salud (OMS) que dio a conocer el Clean Air Institute (Instituto de Aire Limpio) en un informe de 2013, en esta ciudad por año, alrededor de 3 mil personas mueren debido a exposición crónica o aguda con aire contaminado, lo que la iguala con Monterrey y solo debajo de la Cd. México.

Guadalajara (Jalisco, México) fue en 2011 la Ciudad Latinoamericana de más de 1 millón de habitantes que encabezó la lista de concentraciones de ozono (69,3 microgramos por metro cúbico); fue segundo lugar en concentraciones de PM10 (partículas suspendidas menores a diez micras) con $70.1 \mathrm{mg} / \mathrm{m} 3$; segundo lugar para dióxido de nitrógeno $(57.2 \mathrm{mg} / \mathrm{m} 3)$ y cuarto lugar para dióxido de azufre $(13.1 \mathrm{mg} / \mathrm{m} 3)$.

Realizando la investigación y recabando información, se tiene que la propuesta para paliar el problema de contaminación del aire sería el crear e implementar un programa de verificación vehicular para la Zona Metropolitana de Guadalajara, que lleve a la evaluación y el generar los datos necesarios en la medición del impacto y comprobar una posible disminución en los indicadores. 


\section{PROYECTO PARA LOS VERIFICENTROS DE JALISCO}

El terreno de mínimo $500 \mathrm{~m}^{2}$, oficinas, área de cobro, sanitarios, cuarto de gases, cuarto de acometido eléctrico, sala de espera, techumbre, fosas de dinamómetro, muros perimetrales, unidades de aire acondicionado, plancha de concreto y cancelería

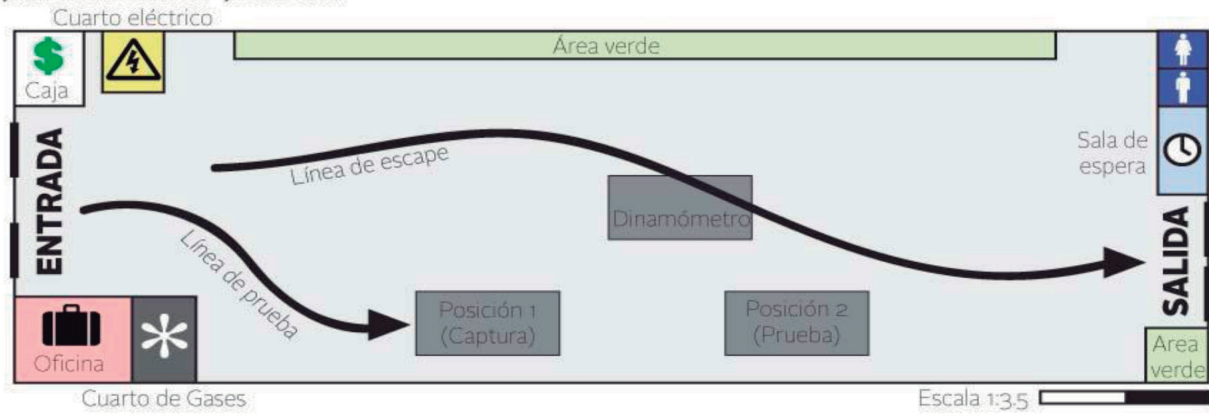

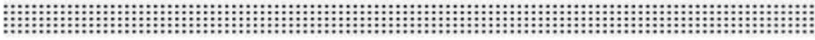

\section{COSTOS DE INVERSIÓN PARA UN CENTRO DE VERIFICACIÓN*}

ARTÍCULOS REQUERIDOS

1 Analizador de gases

1 Dinamómetro

1 Estación meteorológica

1 Centro de cómputo

1 Red de gases

1 Sistema de video

2 Cámaras de video

1 Monitor

1 Procesador de imágenes

1 Sistema de aforo

10 bra civil del verificentro

1 Permisos, estudios y proyectos

10 tros equipos (compresor de aire sin lubricación, unidad de generación de aire cero, etc)

TOTAL inversión estimada:

tpara una sola linea, calculados a precios de may Fuente: estudio del Instituto Mexicano del Petróleo, informe de mayo de 2015

\section{COSTO}

240 mil pesos

315 mil pesos

3,750 pesos

52,500 pesos

40 mil pesos

90 mil pesos

10,600 pesos

2.250 pesos

22,500 pesos

37,500 pesos

875,000 pesos

45 mil pesos 


\section{Esquema 3}

\begin{tabular}{|c|c|c|c|c|}
\hline \multicolumn{5}{|c|}{$\begin{array}{l}\text { Conceptos: separar la causa efecto, usar frases sencillas y breves, eliminar múltiples } \\
\text { objetivos. }\end{array}$} \\
\hline $\begin{array}{l}\text { Cual es la finalidad } \\
\text { del proyecto? } \\
\text { (beneficios, impacto } \\
\text { a nivel sectorial) }\end{array}$ & $\begin{array}{l}\text { Fin: Jalisco Sustentable } \\
\text { ZMG. }\end{array}$ & $\begin{array}{l}\text { Indicadores: } \\
\text { erradicar la } \\
\text { contaminación por } \\
\text { Co2 al 100\% 2016- } \\
2018 .\end{array}$ & $\begin{array}{l}\text { Medios de } \\
\text { verificación: } \\
\text { estadísticas, recibos, } \\
\text { facturas. paginas } \\
\text { transparencia. }\end{array}$ & $\begin{array}{l}\text { Supuestos : Mejora } \\
\text { de la calidad de vida } \\
\text { en la sociedad }\end{array}$ \\
\hline $\begin{array}{l}\text { Por que se lleva a cabo } \\
\text { el proyecto? Impacto } \\
\text { directo, resultadode } \\
\text { utilizar los } \\
\text { componentes) }\end{array}$ & $\begin{array}{l}\text { Propósito: reducir la } \\
\text { emisión de Co2 en el } \\
\text { municipio de GDL- } \\
\text { Tlaquepaque-Salto }\end{array}$ & $\begin{array}{l}\text { Reducir emisión de } \\
\text { Co2 en un } 30 \% \text { en } 1 \\
\text { año en GDL- } \\
\text { Tlaquepaque-Salto }\end{array}$ & $\begin{array}{l}\text { SENER SEMARNAT, } \\
\text { SEMADET, SEFIN, } \\
\text { Ong's, Auditoria } \\
\text { Superior, Camara Dip. } \\
\text { CCE. }\end{array}$ & $\begin{array}{l}\text { Campaña informativa } \\
\text { deeencientización para } \\
\text { verificación vehicular } \\
\text { en la ZMG población } \\
\text { en general. }\end{array}$ \\
\hline $\begin{array}{l}\text { Que debe ser } \\
\text { producido por el } \\
\text { proyecto? }\end{array}$ & $\begin{array}{l}\text { Componentes: Centros } \\
\text { de verificación de } \\
\text { crrisiones Gú y } \\
\text { Capacitación continua. }\end{array}$ & $\begin{array}{l}\text { Unidades } 100 \% \\
\text { Acreditadas como } \\
\text { Vereffeentroencl } \\
\text { primer año. }\end{array}$ & $\begin{array}{l}\text { SENER SEMARNAT, } \\
\text { SEMADET, SEFIN, } \\
\text { Ong's, Auditeria } \\
\text { Superior, Camara Dip. } \\
\text { CCE. }\end{array}$ & $\begin{array}{l}\text { Dotar de equipo a los } \\
\text { talleres acreditados y } \\
\text { eetpacitar. Campaña de } \\
\text { verificación vehicular } \\
\text { en todos los niveles de } \\
\text { transporte. }\end{array}$ \\
\hline $\begin{array}{l}\text { Como se producirán } \\
\text { los componentes? }\end{array}$ & $\begin{array}{l}\text { Actividades: } \\
\text { Implementación de } 5 \\
\text { Centros, con equipo y } \\
\text { sistema de verificación } \\
\text { de emisiones Co2, así } \\
\text { como Formación técnica. }\end{array}$ & $\begin{array}{l}\text { Inversion de } \\
20^{\prime} 000,000.00 \text { en } \\
2016\end{array}$ & $\begin{array}{l}\text { SENER SEMARNAT } \\
\text { SEMADET, SEFIN, } \\
\text { Ong s, Auditoria } \\
\text { Superior, Cámara DIpp. } \\
\text { CCE. }\end{array}$ & $\begin{array}{l}\text { Eficiencia y eficacia en } \\
\text { la aplicación y } \\
\text { comprobación del } \\
\text { presupuesto ejercido } \\
\text { (año fiscal). }\end{array}$ \\
\hline
\end{tabular}

Fuente: Elaboración propia. 2016

\section{Rentabilidad del proyecto}

Según lo requerido por el Instituto Mexicano del Petróleo (2016), establece invertir según sus cálculos $\$ 3$ millones 864 mil por línea o Centro de Verificación.

- $\quad$ Autos en ZMG alrededor de 2 millones 18 mil unidades.

- 3 millones de pesos en infraestructura (500 metros cuadrados).

- 864 mil pesos en equipamiento y tecnología, para una 1 línea.

- Costo $\$ 420$ pesos por auto. Se estarían verificando 200 autos por semana, proyectando 8 mil 800 verificados al año, el retorno de inversión a 18 meses sería de $46.6 \%$

- Por lo que se generarían \$3 millones 696 mil anuales, a 18 meses nos da una cantidad de \$5 millones 544 mil pesos, alcanzando con esto en el mes trece la recuperación de la inversión.

- $\quad$ En diez años generaría ingresos por 37 millones de pesos. 


\section{Conclusiones}

Dadas las proyecciones, se establece que el proyecto es viable y resulta rentable para su operación. Se deberá tener en cuenta que en su implementación el modelo de negocios será decisión del Poder Ejecutivo. Dicho programa de verificación vehicular, sus espacios e instalaciones pueden ser llevados por el estado en su totalidad o, también podría contemplarse la participación de la iniciativa privada. Ahora bien, se especifica el año y periodo de estudio porque fue la última vez que se propuso una estrategia conjunta, del 2018 a la fecha ya no se contempló la posibilidad de llevarla a cabo por parte del nuevo gobierno y la iniciativa privada.

Debe tomarse en cuenta que, en el mes de julio de 2015, se contempla por parte del Instituto Nacional Electoral el proceso de elecciones federal y local, por lo que para el uso de los recursos económicos deberían contemplar el consenso de las fuerzas políticas y predominar los intereses de la sociedad por encima de los particulares partidistas.

\section{Referencias}

Instituto Mexicano del Petróleo [IMP]. (2016). Informe de Autoevaluación Institucional. Ejercicio 2015. Recuperado de https:/www.imp.mx/transparencia/Inf_autoevaluacion.pdf

OBS Business School. (s/f). El marco lógico en la gestión de un proyecto, una herramienta esencial. Universitat de Barcelona. Recuperado de https://obsbusiness. school/es/blog-project-management/herramientas-esenciales-de-un-project-manager/el-marco-logico-en-la-gestion-de-un-proyecto-una-herramienta-esencial

Organización de las Naciones Unidas para la Educación, la Ciencia y la Cultura [UNESCO]. (2017). Expresiones culturales. Árbol de problemas. Recuperado de http:// www.unesco.org/new/es/culture/themes/\%20cultural-diversity/diversity-of-cultural\%20expressions/tools/policy-guide/planificar/diagnosticar/arbol-de-problemas/ 